Arq. Bras. Med. Vet. Zootec., v.70, n.1, p.57-63, 2018

\title{
Linfoma mediastinal em equino - relato de caso
}

\author{
[Equine mediastinal lymphoma - case report] \\ K. Gravena ${ }^{1}$, G.G. Rivera ${ }^{1}$, R.A. Navarrete ${ }^{1}$, P.C. Jark $^{1}$, A.A. Camacho ${ }^{2}$, \\ J.C. Lacerda-Neto ${ }^{2}$ \\ ${ }^{1}$ Aluno de pós-graduação - Universidade Estadual Paulista Júlio de Mesquita Filho - Unesp - Jaboticabal, SP \\ ${ }^{2}$ Universidade Estadual Paulista Júlio de Mesquita Filho - Unesp - Jaboticabal, SP
}

\section{RESUMO}

Uma égua SRD, com 20 anos de idade, foi encaminhada ao hospital veterinário com histórico de emagrecimento, perda de apetite e aumento de volume na região peitoral e no pescoço. Aos exames físico e laboratorial, constatou-se que o animal apresentava taquicardia, taquipneia, hiperproteinemia, hiperfibrinogenemia e hipoalbuminemia. À auscultação cardíaca, os sons das válvulas pulmonar e aórtica não foram audíveis. Realizaram-se as avaliações eletrocardiográfica e ecodopplercardiográfica transtorácica bilateral, nas quais se observaram presença de efusão pleural e deslocamento caudal do coração. Na varredura ultrassonográfica ao redor do coração, constatou-se a presença de uma massa na base do coração. Para tentar amenizar o quadro respiratório, foi realizada drenagem torácica. A efusão pleural apresentava característica serossanguinolenta, com padrão citológico inflamatório. Algumas horas após retornar a baia, o animal veio a óbito. Realizou-se a necropsia, na qual pôde ser observada a presença da massa posicionada ao redor do tronco braquiocefálico. $\mathrm{Na}$ análise histopatológica, diagnosticou-se o linfoma difuso de células pequenas e médias.

Palavras-chave: neoplasia hematopoiética, ecodopplercardiografia, cavalo

\begin{abstract}
A 20-year-old mixed breed mare was referred to the veterinary hospital with history of weight loss, loss of appetite, and swelling of the chest and neck. On physical exam the patient was in tachycardia and tachypnea and laboratory results indicated hyperproteinemia, hyperfibrinogenemia and hypoalbuminemia. On cardiac auscultation, the pulmonary and aortic valves sounds were not audible. Presence of pleural effusion and heart caudal displacement were identified on electrocardiographic and Doppler echocardiography evaluation and a mass at the base of the heart was oberved through ultrasound scanning around the heart. To alleviate the respiratory condition, thoracic drainage was performed. The pleural effusion presented characteristic serosanguineous with cytologic inflammatory. After returning to stall, the animal died. In necropsy, the presence of a mass positioned around the brachiocephalic trunk was identified and histopathology results were compatible with diffuse small and medium cell lymphoma.
\end{abstract}

Keywords: hematopoietic neoplasms, doppler echocardiography, horse

\section{INTRODUÇ̃̃o}

O linfoma, também conhecido por linfoma maligno ou linfossarcoma, é a neoplasia hematopoiética mais comumente encontrada em equinos (Mair et al., 1985; Meyer et al., 2006; Muñoz et al., 2009; Durham et al., 2012), no entanto é considerado raro quando se compara sua ocorrência em outras espécies (Taintor e

Recebido em 10 de setembro de 2016

Aceito em 12 de abril de 2017

E-mail: kamilagravena@yahoo.com.br
Schleis, 2011). Esse tumor tem sido classificado em multicêntrico ou generalizado, alimentar, mediastinal, cutâneo (Mair et al., 1985; Mair e Brown, 1993; Muñoz et al., 2009) e isolado (Muñoz et al., 2009; Taintor e Schleis, 2011). O linfoma nessa espécie é uma doença de caráter heterogêneo quanto à variabilidade de sinais clínicos, às alterações laboratoriais e aos achados patológicos (Mair et al., 1985; Platt, 1988; Meyer et al., 2006). Segundo Durham et al. 
(2012), ao avaliarem 203 casos de linfoma, 41\% eram multicêntricos, $19 \%$ cutâneos, $11 \%$ alimentares e apenas 4,5\% mediastinais; outras localizações isoladas, como órbita ocular, baço, cavidade nasal, sistema nervoso central, cavidade oral, fígado, medula óssea e coração, somavam $18,5 \%$, e $6 \%$ das localizações não foram identificadas.

Os sinais clínicos manifestados no início do processo são inespecíficos, porém, com a progressão da doença, os sinais passam a refletir a disfunção do órgão acometido (Mair et al., 1985; Taintor e Schleis, 2011). Apesar disso, alguns sinais são comuns a todas as formas de linfoma equino e incluem perda de peso, depressão, letargia, edema da porção ventral do corpo ou parte distal do membro e febre recorrente (Mair e Brown, 1993; Meyer et al., 2006; Muñoz et al., 2009; Taintor e Schleis, 2011).

O linfoma de mediastino pode também ser denominado linfoma torácico ou de timo (Muñoz et al., 2009; Taintor e Schleis, 2011). Esta é a neoplasia mais comum do tórax dos equinos e pode ser observada em todas as raças, idades e sexo (Mair et al., 1985; Mair e Brown, 1993; Meyer et al., 2006). A evolução da doença é muito rápida e, em geral, ocorre no intervalo de uma a oito semanas após o aparecimento dos primeiros sinais (Mair et al., 1985). Cavalos com a forma mediastinal também podem apresentar intolerância ao exercício, dispneia, tosse, edema ventral (especificamente peitoral) e distensão da veia jugular (Mair et al., 1985; Mair e Brown, 1993). Na auscultação torácica, pode-se observar o abafamento das bulhas e, na percussão torácica e no exame ultrassonográfico, pode ser detectada evidência de efusão pleural (Taintor e Schleis, 2011).

Em geral, o diagnóstico ocorre tardiamente, já que a doença possui natureza insidiosa e não apresenta sinais clínicos patognomônicos (Taintor e Schleis, 2011). A abordagem do equino com suspeita de linfoma inicia-se com exame físico, palpação retal, hemograma completo e exames bioquímicos. As avaliações ultrassonográficas do tórax e do abdômen ajudam na localização da massa e na determinação da extensão do comprometimento do órgão acometido (Muñoz et al., 2009; Taintor e Schleis, 2011). A aspiração ou biópsia de linfonodos ou massas é ideal para o diagnóstico, no entanto pode ser de difícil acesso no caso de neoplasias dentro do tórax ou do abdômen (Taintor e Schleis, 2011). O diagnóstico diferencial pode ser realizado por meio de avaliação citológica da efusão pleural após a toracocentese (Meyer et al., 2006). Essa efusão se apresenta como transudato modificado contendo níveis baixos de proteína e predomínio de células linfoides, das quais muitas com formas bizarras e com figuras mitóticas (Mair et al., 1985).

O prognóstico de cavalos com linfoma depende do local acometido e da fase de evolução da doença, porém, na maioria das vezes, o animal vai a óbito (Taintor e Schleis, 2011). Os poucos relatos de tratamentos de cavalos com linfomas reportam o uso de quimioterápicos (Saulez et al., 2004) assim como de radioterapia (Henson et al., 2004).

Diante do exposto, o objetivo deste trabalho foi relatar um caso de linfoma localizado na base do coração em uma égua.

\section{CASUÍSTICA}

Uma égua, SRD, com 20 anos de idade, foi encaminhada ao Hospital Veterinário "Governador Laudo Natel" com histórico de emagrecimento, perda de apetite e aumento de volume na região peitoral e no pescoço com evolução de 20 dias (Fig. 1). Aos exames físico e laboratorial, constatou-se que o animal apresentava taquicardia (48bpm), taquipneia (20mpm), hiperproteinemia $(8,2 \mathrm{~g} / \mathrm{dL})$, hiperfibrinogenemia $(800 \mathrm{mg} / \mathrm{dL})$ e hipoalbuminemia $(1,71 \mathrm{~g} / \mathrm{dL})$. Também foram observados aumento do aspartato aminotransferase (AST) (606U/L), gamaglutamiltranferase (GGT) (91U/L) e fosfatase alcalina (FA) (845U/L). No exame hemogasométrico, observou-se a pressão parcial de oxigênio $\left(\mathrm{PO}_{2}\right)$ de $41,7 \mathrm{mmHg}$, pressão parcial de dióxido de carbono $\left(\mathrm{PCO}_{2}\right)$ de $36,5 \mathrm{mmHg}$, o excesso/déficit de base (EB) de $-1,45 \mathrm{mmol} / \mathrm{L}$, a saturação de oxigênio $\left(\mathrm{SO}_{2}\right)$ de $73,4 \%$, o logaritmo negativo da atividade de íons hidrogênio $(\mathrm{pH})$ de 7,394, as concentrações de bicarbonato $\left(\mathrm{HCO}_{3}{ }^{-}\right)$de $23,0 \mathrm{mmol} / \mathrm{L}$, íon sódio $\left(\mathrm{Na}^{+}\right) \quad 134,7 \mathrm{mmol} / \mathrm{L}$, íon potássio $\left(\mathrm{K}^{+}\right)$ $3,85 \mathrm{mmol} / \mathrm{L}$, cálcio ionizado (Cai) $1,457 \mathrm{mmol} / \mathrm{L}$, glicose $3,6 \mathrm{mmol} / \mathrm{L}$ e lactato de $5,6 \mathrm{mmol} / \mathrm{L}$. 

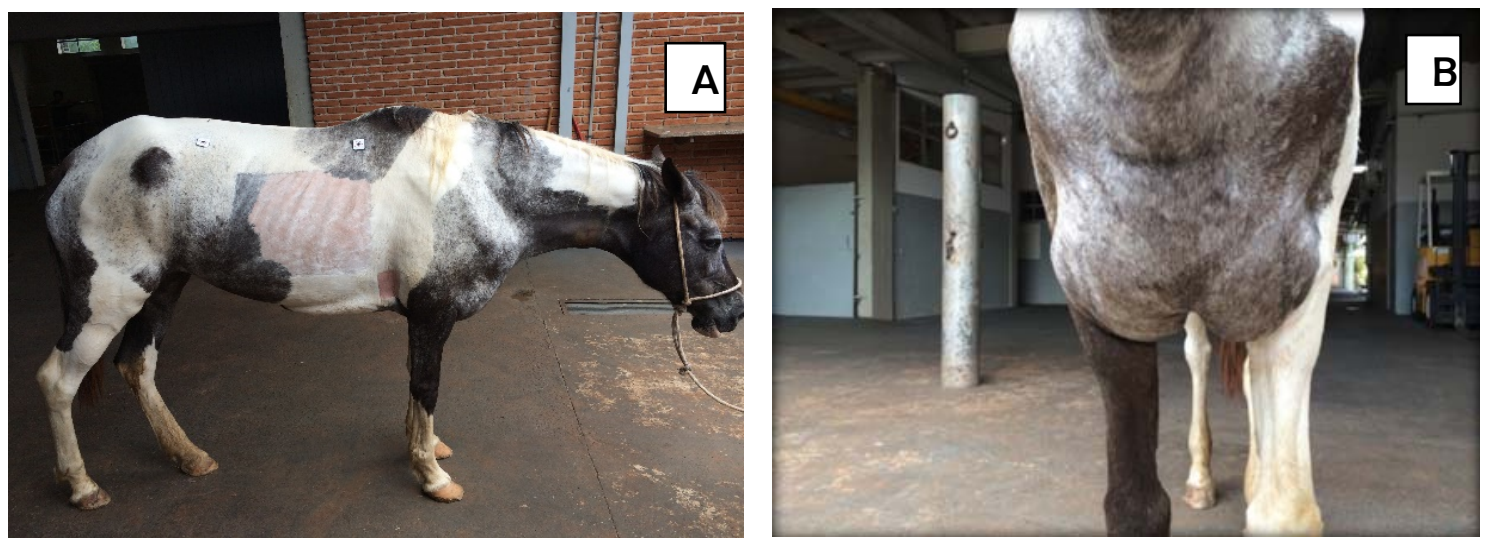

Figura 1. A) Égua, SRD, apresentando emagrecimento e apatia, com aumento de volume na região peitoral e no pescoço. B) Edema peitoral cranioventral.

À auscultação cardíaca, os sons das válvulas pulmonar e aórtica não foram audíveis e à auscultação pulmonar observaram-se murmúrios vesiculares na região dorsal e silêncio na porção ventral. Realizou-se a avaliação eletrocardiográfica (ECG PC - TEB, Brasil) na derivação base-ápice, observando-se taquicardia sinusal de 53bpm (Tab. 1). Em seguida, efetuou-se o exame ecodopplercardiografico transtorácico bilateral (MyLab 30 Vet Gold, Esaote, Itália ) com transdutor de $1-4 \mathrm{MHz}$ (PA 240, Esaote, Itália ) (Tab. 2), no qual se observou a presença de efusão pleural e deslocamento caudal do coração. Essa condição impossibilitou a realização de todas as medidas. Devido à alteração da posição cardíaca, foi realizada varredura ultrassonográfica ao redor do coração, no qual se observou presença de fibrina e de uma massa hipervascularizada na base do coração (Fig. 2A, $\mathrm{B}$ e C).

No dia seguinte, as frequências cardíaca e respiratória aumentaram, chegando a $60 \mathrm{bpm}$ e $32 \mathrm{mpm}$, respectivamente. A mucosa ocular apresentava-se congesta. Nova ultrassonografia torácica e abdominal foi realizada, verificando-se expansão de volume de efusão pleural e leve ascite.

Exames laboratoriais, realizados no terceiro dia de internamento, revelaram os seguintes valores para creatinina $(3,1 \mathrm{mg} / \mathrm{dL})$, ureia $(94 \mathrm{mg} / \mathrm{dL})$, AST (754U/L), GGT (153U/L), bilirrubina total $(2,56 \mathrm{mg} / \mathrm{dL})$, bilirrubina direta $(0,56 \mathrm{mg} / \mathrm{dL}), \mathrm{FA}$ $(1459 \mathrm{U} / \mathrm{L})$ e albumina $(1,82 \mathrm{~g} / \mathrm{dL})$. No exame hemogasométrico, a $\mathrm{PO}_{2}$ diminuiu para $37,7 \mathrm{mmHg}$, a $\mathrm{PCO}_{2}$ para $30,2 \mathrm{mmHg}$, o EB para $10,29 \mathrm{mmol} / \mathrm{L}$, a $\mathrm{SO}_{2}$ para $61,0 \%$, o $\mathrm{pH}$ para 7,268 , as concentrações de $\mathrm{HCO}_{3}^{-}$também diminuíram para $15,6 \mathrm{mmol} / \mathrm{L}, \mathrm{Na}^{+} 115,7 \mathrm{mmol} / \mathrm{L}$ e o Cai $1,333 \mathrm{mmol} / \mathrm{L}$. A concentração de $\mathrm{K}^{+}$ aumentou para $4,50 \mathrm{mmol} / \mathrm{L}$, assim como a glicose para $6,1 \mathrm{mmol} / \mathrm{L}$ e o lactato para $7,5 \mathrm{mmol} / \mathrm{L}$. Associado a essas alterações, houve aumento da FC (80bpm) e FR (36mpm), a mucosa ocular manteve-se congesta, o tempo de preenchimento capilar estava aumentado (quatro segundos) e a respiração tornou-se abdominal. Para tentar amenizar o quadro respiratório, foi realizada drenagem torácica direita e esquerda, com cateter de Foley, no qual foram extraídos 7 e 6,5 litros de cada um dos lados, respectivamente. A efusão pleural apresentava característica serossanguinolenta, com padrão citológico inflamatório, presença de hemácias, alguns agregados plaquetários, fibrina, eritrofagocitose, poucos neutrófilos discretamente degenerados, raras células mesoteliais reativas, grandes quantidades de macrófagos hiperativos e raríssimos linfócitos com critérios cariocitoplasmáticos discretamente suspeitos. 


\section{Gravena et al.}

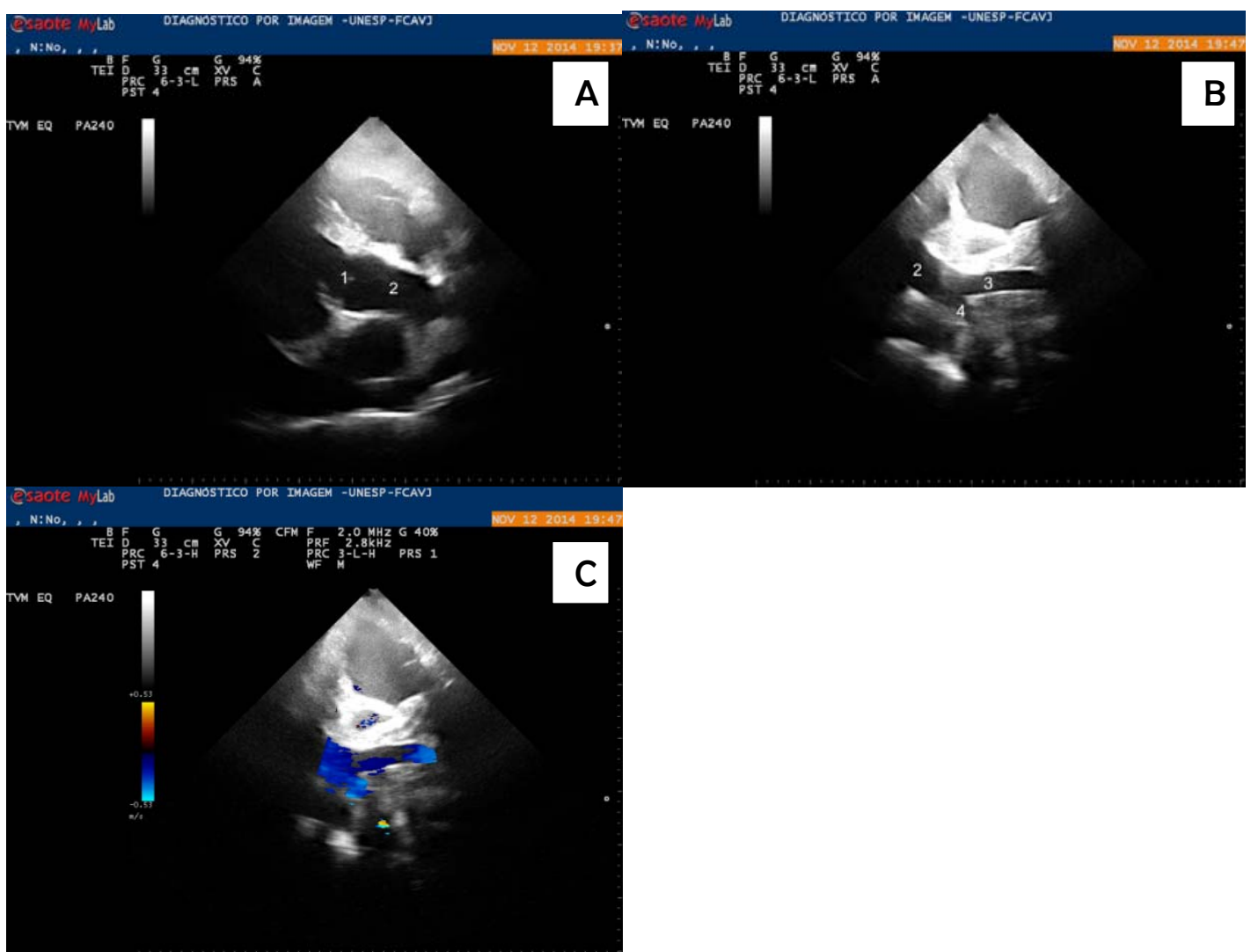

Figura 2. A) Imagem ecocardiográfica longitudinal cinco câmaras via de saída do ventrículo esquerdo, válvula aorta (1), tronco aórtico (2). B) Continuação da imagem ecocardiográfica longitudinal cinco câmaras, evidenciando o tronco braquiocefálico (3) e aorta descendente (4). C) Doppler color (azul) do fluxo laminar aórtico.

Após a drenagem pleural, foram repetidos os exames de eletrocardiografia e ecodopplercardiografia, obtendo-se no ECG taquicardia sinusal (81bpm) e aumento nas amplitudes de onda $\mathrm{P}$ e onda $\mathrm{R}$ ao ser comparado com o exame anterior, além de o segmento ST se apresentar supradesnivelado (Tab. 1). $\mathrm{Na}$ ecodopplercardiografia, pôde ser observada ainda a presença de efusão e a permanência do posicionamento cardíaco alterado (Tab. 2). A respiração apresentava-se um pouco mais torácica, porém o animal apresentava-se apático, com taquipneia e taquicardia. Algumas horas após retornar a baia, o animal veio a óbito.

Tabela 1. Valores eletrocardiográficos obtidos antes e após toracocentese

\begin{tabular}{lcc} 
& Antes & Após \\
\hline Frequência Cardíaca (bpm) & 53 & 81 \\
Comprimento de onda P (seg) & 0,107 & 0,120 \\
Amplitude de onda P (mV) & 0,109 & 0,234 \\
Intervalo PR (seg) & 0,227 & 0,167 \\
Comprimento de QRS (seg) & 0,107 & 0,140 \\
Amplitude de onda R (mV) & 0,656 & 1,500 \\
Segmento ST & Isoelétrico & Supradesnivelado \\
Intervalo QT (seg) & 0,440 & 0,353 \\
Onda T & Positiva $>25 \%$ de R & Positiva $>25 \%$ de R
\end{tabular}


Tabela 2. Valores ecocardiográfico obtidos em modo $\mathrm{M}$ antes e após toracocentese

\begin{tabular}{lcc}
\hline Modo M & Antes & Após \\
\hline RVd (mm) & 8,9 & 10,3 \\
IVSd (mm) & 22,1 & 45,9 \\
LVd (mm) & 42,8 & 52,8 \\
PWd (mm) & 35,4 & 42,5 \\
IVSs (mm) & 36,9 & 56,2 \\
LVs (mm) & 16,2 & 32,1 \\
PWs (mm) & 35,4 & 51,6 \\
FS $(\%)$ & 62 & 39 \\
\hline
\end{tabular}

Realizou-se a necropsia, na qual pôde ser observada a presença da massa cranial ao coração com tamanho aproximado de 12 x 15 x $17 \mathrm{~cm}$. Essa massa estava posicionada próximo ao tronco aórtico, mais especificamente ao redor do tronco braquiocefálico (Fig. 2D). Os linfonodos mediastinais apresentavam a mesma consistência da massa, porém o tamanho não estava alterado. Foram coletados fragmentos dessa massa para a realização do histopatológico, os quais foram processados e avaliados pela técnica de coloração rotineira de hematoxilina e eosina (HE).

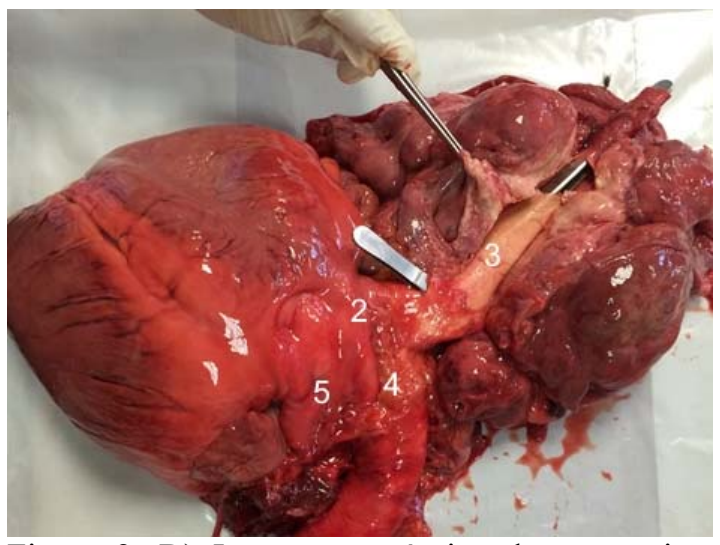

Figura 2. D) Imagem anatômica de necropsia, fase esquerda do coração e grandes vasos, tronco aórtico (2) e tronco pulmonar (5). Observa-se que o tronco braquiocefálico (3) se apresenta circundado pela neoplasia descrita.

O resultado da análise tecidual revelou a presença de neoplasia maligna invadindo difusamente tecido fibroadiposo, caracterizado por proliferação linfoide monótona, caracterizada por células redondas pequenas e médias, de aspecto plasmocitoide, com núcleos hipercorados, alguns clivados, periféricos, e citoplasma moderado eosinofílico.
Tais achados permitiram concluir o diagnóstico de linfoma difuso de células pequenas e médias.

\section{DISCUSSÃO}

O curso clínico da doença é considerado rápido. Mair et al. (1985), ao avaliarem 11 equinos, observaram o intervalo de uma a oito semanas entre o aparecimento dos sinais e a eutanásia. Já Muñoz et al. (2009) descrevem ser uma doença na qual os sinais progridem ao longo de semanas a meses, embora possam apresentar início súbito. No caso acima relatado, o aparecimento dos sinais ocorreu 20 dias antes de o animal ser encaminhado ao hospital veterinário, ocasião em que permaneceu internado por três dias e veio a óbito, totalizando três semans e dois dias de intervalo.

Os sinais clínicos de emagrecimento, inapetência, perda de apetite, edema ventral (principalmente na região peitoral e no pescoço) e efusão pleural também foram observados nos casos relatados por Mair et al. (1985). Dos três casos relatados por De Clercq et al. (2004), todos apresentaram o edema ventral, porém apenas um demonstrou depressão e anorexia. Meyer et al. (2006) examinaram 37 equinos, dos quais 25 exibiram perda de peso e 21 apresentaram edema. Durham et al. (2012) avaliaram 109 casos; desse total, 30\% (32 animais) foram descritos com perda de peso, anorexia e depressão e 18\% (20 animais) com edema ventral. Segundo Muñoz et al. (2009), além desses sinais, a linfoadenopatia regional também é um dos principais sinais, sendo observado em nove dos 37 equinos avaliados por Meyer et al. (2006).

O diagnóstico do linfoma em equinos é realizado tardiamente, pois essa doença não apresenta sinal patognomônico (Taintor e Schleis, 2011). No entanto, o diagnóstico pode ser realizado ante mortem, por meio de exames ultrassonográficos que localizam a massa (De Clercq et al., 2004) ou por aspirado/biópsia do linfonodo afetado ou da massa, o que se torna difícil quando a neoplasia encontra-se dentro do tórax (Taintor e Schleis, 2011).

Nos cães e gatos, o linfoma é considerado uma neoplasia altamente esfoliativa. Segundo Davies e Forrester (1996), 79\% dos casos de linfoma mediastinal em felinos foram diagnosticados por 
meio da análise citológica da efusão pleural. Nos equinos, o comportamento dessa neoplasia parece ser menos esfoliativo, uma vez que, no estudo de Mair e Brown (1993), apenas 14 dos 38 casos de linfoma mediastinal foram diagnosticados pelo exame citológico do fluido coletado da cavidade torácica.

No presente relato, o diagnóstico baseou-se no exame ecodopplercardiográfico transtorácico, o qual possibilitou a observação do deslocamento cardíaco, e com a varredura ultrassonográfica, visualizou-se a massa na base cardíaca, assim como descrito por De Clercq et al. (2004), que, além do deslocamento caudal cardíaco, também relatam o deslocamento dorsal dos pulmões. $\mathrm{Na}$ tentativa de confirmar o diagnóstico, efetuou-se a avaliação da efusão pleural, a qual se apresentou serossanguinolenta, como exposto por De Clercq et al. (2004). Na avaliação celular, foram observados raríssimos linfócitos com critérios cariocitoplasmáticos discretamente suspeitos, semelhante aos dados encontrados em dois animais descritos por De Clercq et al. (2004), porém o presente relato difere do terceiro caso descrito por De Clercq et al. (2004), os quais demonstraram presença incomum de células neoplásicas, com linfoblastos e linfócitos com núcleos irregulares e figuras mitóticas.

$\mathrm{Na}$ necropsia, a massa apresentou tamanho aproximado de 12 x 15 x $17 \mathrm{~cm}$ e estava posicionada ao redor do tronco braquiocefálico, no entanto essa neoplasia pode ter diversos tamanhos e localizações. Segundo Mair et al. (1985), ao avaliarem 11 animais após a morte com linfossarcoma de cavidade torácica, verificou-se que os tamanhos variaram de 14 a $25 \mathrm{~cm}$ de diâmetro e a maioria apresentava-se na porção mediastinal anterior. Nos três casos relatados por De Clercq et al. (2004), o primeiro apresentou $17 \mathrm{~cm}$ de diâmetro e sua localização era ao redor do nervo laríngeo esquerdo; o segundo e o terceiro caso estavam no mediastino cranial com 8 e $7 \mathrm{~cm}$ de diâmetro, respectivamente. Platt (1988) não relata o tamanho, no entanto ele descreve um caso em que os linfonodos mediastínico, bronquial e cervical caudal profundo aumentaram, formando uma massa que recobriu o coração e envolveu grandes vasos, com invasão da traqueia e do esôfago.
O exame histopatológico do presente relato revelou o linfoma difuso de células pequenas e médias, classificação esta que não se enquadra nos padrões da Organização Mundial da Saúde (OMS), a qual se baseia na imunofenotipagem. Entre 187 casos de linfoma equino classificados pela OMS, 87 casos (43\%) foram do subtipo de linfoma de grandes células $\mathrm{B}$ rico em células $\mathrm{T}$ (Durham et al., 2012). Essa forma de linfoma pode ser de difícil classificação no exame citológico da efusão pleural ou no exame histopatológico, uma vez que apresenta população de linfócitos neoplásicos associados a linfócitos maduros, caracterizando uma população heterogênea. Diferentemente de cães em que a realização do exame imunohistoquímico apresenta valor prognóstico, sendo os linfomas de alto grau de imunofenótipo $\mathrm{T}$ associados a menores taxas de sobrevida, não existem dados a respeito da implicação prognóstica do imunofenótipo em equinos com linfoma, portanto, devido à ausência de aplicação prognóstica associada a questões inerentes ao proprietário, o exame imuno-histoquímico não foi realizado nesse caso.

Embora o linfoma seja considerado uma das neoplasias mais responsivas à quimioterapia em cães, gatos e seres humanos, essa modalidade terapêutica não é amplamente utilizada em equinos, existindo poucos dados na literatura a respeito da eficácia e das taxas de resposta em cavalos. A evolução desfavorável rápida do quadro do animal do presente relato, associada à falta de diagnóstico ante mortem, impossibilitou a realização de um tratamento mais direcionado para o paciente em questão.

\section{REFERÊNCIAS}

DAVIES, C.; FORRESTER, S.D. Pleural effusion in cats:82 cases (1987 to 1995). J. Small Anim Pract., v.37, p.217-224, 1996.

DE CLERCQ, D.; VAN LOON, G.; LEFÈRE, L. et al. Ultrasound-guided biopsy as a diagnostic aid in three horses with a cranial mediastinal lymphosarcoma. Vet. Rec., v.154, p.722-726, 2004.

DURHAM, A.C.; PILLITTERI, C.A.; SAN MYINT, M. et al. Two hundred three cases of equine lymphoma classified according to the World Health Organization (WHO) classification criteria. Vet. Pathol., v.50, p.86-93, 2012. 
HENSON, F.M.D.; DIXON, K.; DOBSON, J.M. Treatment of 4 cases of equine lymphoma with megavoltage radiation. Equine Vet. Educ., v.16, p.312-314, 2004.

MAIR, T.S.; BROWN, P.J. Clinical and pathological features of thoracic neoplasia en the horse. Equine Vet. J., v.25, p.220-223, 1993.

MAIR, T.S.; LANE, J.G.; LUCKE, V.M. Clinicopathological features of lymphosarcoma involving the thoracic cavity in the horse. Equine Vet. J., v.17, p.428-433, 1985.

MEYER, J.; DELAY, J.; BIENZLE, D. Clinical, laboratory, and histopathologic features of equine lymphoma. Vet. Pathol., v.43, p.914-924, 2006.
MUÑOZ, A.; RIBER, C.; TRIGO, P. et al. Hematopoietic neoplasias in horses: myeloproliferative and lymphoproliferative disorders. J. Equine Sci., v.20, p.59-72, 2009.

PLATT, H. Observations on the pathology of non-alimentary lymphomas in the horse. $J$. Comp. Pathol., v.98, p.177-194, 1988.

SAULEZ, M.N.; SCHLIPF JR, J.W.; CEBRA, C.K. et al. Use of chemotherapy for treatment of a mixed-cell thoracic lymphoma in a horse. $J$. Am. Vet. Med. Assoc., v.224, p.733-738, 2004.

TAINTOR, J.; SCHLEIS, S. Equine lymphoma. Equine Vet. Educ., v.23, p.205-213, 2011. 